\title{
The Use of Donor Corneas Which Have Undergone Refractive Surgery in Keratoplasty
}

\author{
Eda Asilyazici, () Banu Acikalin, () Murat Garli, () Yildirim Kocapinar \\ Department of Ophthalmology, Fatih Sultan Mehmet Training and Research Hospital, Istanbul, Turkey
}

\begin{abstract}
Due to the rapid developments in techniques and devices used in refractive surgery, these surgeries are applied to more than before, and different techniques are emerging every day. Laser in situ keratomileusis, photorefractive keratectomy (PRK), and RK are the procedures performed most frequently. Eye Bank Association of America Medical Standards does not permit corneas from patients who have undergone refractive procedures to be used in penetrating keratoplasty, anterior lamellar keratoplasty, or tectonic grafting procedures. However, these standards allow these corneas for endothelial keratoplasty. According to these standards identification of these corneas is critical and studies for identification methods are going on. Several case reports have highlighted the difficulty in using post-refractive surgery corneas in penetrating keratoplasty. However, these corneas have been used successfully for endothelial keratoplasty with equivalent outcomes in topography, endothelial cell count, and visual acuity. The increase in endothelial keratoplasty procedures and the successful results of endothelial keratoplasty with post-refractive corneas are promising.
\end{abstract}

Keywords: Donor cornea, endothelial keratoplasty, refractive surgery.

\section{Introduction}

Cornea is one of the most common transplanted tissue and keratoplasty is among the most successful transplantation surgeries. Corneal tissue that is distorted and lost its function is replaced with healthy corneal tissue. Nowadays, corneal transplantation can be performed targeting only the diseased corneal layer. Corneal blindness is the third reason of blindness after cataract and glaucoma, and blindness due to bilateral cornea diseases is present in nearly 10 million people worldwide (I, 2).

In 1944, the American ophthalmologist R. Townley Paton suggested that corneal supply should be provided by a more legal and regular system, and the first Eye-Bank for Sight Restoration in the world was established in New York (3). It is aimed that the cornea is provided, stored, and distributed to the transplantation surgeons through the establishment of the eye bank. Thus, the corneas obtained individually and uncontrolled will be collected by a center and collected, evaluated and distributed in a healthy manner. After the establishment of the first cornea bank, corneal banks developed and increased in number. Corneal transplant criteria are constantly being updated, and efforts are made to standardize these criteria. As with organ donation, tissue donation is still not at the point, where it is desired to reach all over the world. According to Ministry of Health data for 2008, about 10,000 people were waiting for corneal tissue transplant in Turkey. With the beginning of corneal transplantation in more centers and benefit of cornea banks, the number of patients waiting for corneal transplantation decreased to 2500 according to Ministry of Health 2015 data. However, according to Ministry of Health data, while the number of corneal transplants performed in Turkey, 1553 in 2010, this

Address for correspondence: Eda Asilyazici, MD. Fatih Sultan Mehmet Eğitim ve Araştırma Hastanesi, 
number increased to 3155 in 2015. The American Eye Bank reported that more than 46.000 donor corneas were needed in 2010 (4). The need for corneal tissue as well as the number of corneal transplants has increased considerably, and many transplantation techniques have been developed. Endothelial keratoplasty is now used as a standard treatment in the patients especially with endothelial layer dysfunction instead of penetrating keratoplasty. Especially endothelial keratoplasty has become a preferred method since the early 2000s. The development of transplantation techniques has enabled the use of corneas that were previously not suitable for transplantation, and with the development of technology, the number of refractive surgeries increased all around the world. This increase shows the need for a detailed examination of the cornea before transplantation.

\section{The Use of Donor Corneas Which Have Undergone Refractive Surgery, As a Graft}

Today, refractive surgeries are among the most frequently performed surgeries. Laser in situ keratomileusis (LASIK) is now a surgical method that can be applied even in children, with certain indications (5). In 2005, the American Eye Bank Association changed its medical standards in light of technological advances in endothelial keratoplasty methods. The new standards of the association do not allow the use of donor corneas with refractive surgery in penetrating keratoplasty, anterior lamellar keratoplasty, epikeratoplasty, or tectonic corneal graft surgery. Provided that posterior stroma and endothelial layer are not affected, and the surgeon is informed before the surgery, donor cornea with a history of anterior corneal stromal disease unrelated to infection can be used in endothelial keratoplasty (6). In patients undergoing penetrating keratoplasty and anterior lamellar keratoplasty, the crucial criterion for preventing poor visual outcome is the very precise determination of donor corneas. Since sufficient information cannot be obtained for safe surgery in postmortem biomicroscopic examination of refractive corneal corneas such as photorefractive keratectomy (PRK) and LASIK, many techniques have been developed for objective evaluation. Because, in most cases, information from the relatives of the cases is not enough and detailed examination of the donor corneas is very important.

Confocal microscopy studies showed long-standing epithelial hyperplasia after myopic PRK and LASIK (7). In other confocal microscopy studies, corneas undergoing LASIK showed microfolds in the Bowman's layer in addition to the deposits at the level of the flap interface (8). In histological studies of LASIK flaps both performed with a microkeratome and femtosecond laser, varying amounts of keratocyte necrosis and fibrous scar formation have been shown at the flap interface (9).
Penetrating keratoplasties which are mistakenly performed with donor corneas that have undergone refractive surgery have been reported, and these cases provided us useful information. These first experiences are very important because nowadays the increase in refractive procedures makes it possible for every corneal surgeon to transplant such a cornea. Michaeli-Cohel et al. (10) reported two penetrating keratoplasty cases with donor corneas that have undergone LASIK. In the first case after placing many of the sutures, it was seen that the graft was separated into two layers outwardly. In the second case (the other pair of the donor cornea used in the first case), corneal edema was noted during trephination, but no outward separation was observed in the graft. The retrospective evaluation showed these corneas have undergone refractive surgery. 6 months after the operation, 6 and 2.5 diopters (D) astigmatism were detected in these patients, respectively. Similarly, Farias et al. reported that penetrating keratoplasties applied to a patient with keratoconus had similar intraoperative outward bilateral cleavage in the graft after mistakenly use of donor corneas with LASIK. Other than that, the surgery was completed without any complications. 6 months after the operation the patient had $3.75 \mathrm{D}$ astigmatism (II).

\section{The Use of Donor Corneas With Anterior Stromal Disturbance}

Due to recent eye bank applications, the use of donor corneas those lost their stromal integrity secondary to stromal scar, pterygium, or refractive surgery is not allowed for penetrating keratoplasty and anterior lamellar keratoplasty. Both these diseases have a negative effect on the final visual acuity of the recipient eye, and the use of these corneas make the surgery more complicated. Endothelial keratoplasty has now begun to be used in the treatment of many corneal diseases previously treated with penetrating keratoplasty. According to the current rates of corneal transplantation, endothelial keratoplasty is approaching to penetrating keratoplasty. According to 2012 records, penetrating keratoplasty was applied to 36.716 cases in the USA, and endothelial keratoplasty was applied in 24.277 cases (I2).

The first study using corneas which have undergone refractive surgery in endothelial keratoplasty was reported in 2007 by Armor et al. (13) In this study, 39 donor corneas which were not suitable for penetrating keratoplasty, were used in deep lamellar endothelial keratoplasty. Before endothelial keratoplasty, out of 39 donors, only one had undergone LASIK and the surgery was successfully performed despite LASIK flap. Although most of these corneas have stromal scarring due to pterygium, no visual impairment developed after the operation. In addition, there was no statistically significant difference in endothelial cell density and surface regularity index. 
Another case-control study was performed by Philips et al. (14) In this study, Descemet's membrane endothelial keratoplasty (DMEK) was performed on $4 \mathrm{I}$ patients with the anterior stromal disease, LASIK and radial keratotomy (RK) operation, stromal scar, and pterygium, which were not considered suitable for penetrating keratoplasty. However, high-grade primary graft rejection was seen. 6 months after the surgeries there was no statistically significant difference between cases and controls in terms of the best visual acuity, endothelial cell count, and topographic measurements such as surface regularity index and surface asymmetry index.

Khalifa et al. (15) reported the results of analysis of donor corneas that had previously undergone RK by making two corneal lenticules with a microkeratome. In one of the corneas, they found a radial endothelium scar originating from the RK incision. In addition, despite the smooth appearance in anterior optic coherence tomography (OCT) and the scanning electron microscope, the presence of epithelial cells in the histological section of the RK incision has been demonstrated. Based on their findings, the authors noted that donor corneas with RK might not be suitable for endothelial transplantation. Later, they reported their endothelial transplantation case series with donor corneas undergone PRK and LASIK surgeries with visual improvement in all patients except one (I6).

Many postmortem methods have been developed to identify and isolate the corneas that have undergone refractive surgery in the light of the complications that develop after their use in anterior and penetrating keratoplasties. While corneas undergone RK surgery can be easily distinguished by biomicroscopy, it is not easy to distinguish corneas undergone PRK and LASIK surgeries. It is important to make a very detailed and precise identification to avoid false discrimination of tissues undergoing refractive surgery. Kang et al. (I7) used two methods of identification and discrimination: Biomicroscopy and patient history. The misdiagnosis rate was $13.5 \%$ with a medical history, and the rate was $18.2 \%$ with biomicroscopy. When both methods are used, the misdiagnosis rate decreases to $3.4 \%$.

However, Due to the lack of patient records and high misdiagnosis rate with the only biomicroscopy, many methods have been studied to identify corneas undergone refractive surgery. Ousley and Terry used Orbscan (Orbtek, Inc., Salt Lake City, Utah, USA) to compare the central and midperipheral pachymetry and curvature measurements to identify corneas that undergone PRK (I8). Later, they continued their studies using a portable topography device. According to the results obtained by portable topography, the curvature measurement differences in corneal zones of 3 and 7 $\mathrm{mm}$ in all LASIK eyes and one of two eyes with hexagonal keratometry was found to be out of \pm 2 standard devia- tions (SD). However, the difference was found within SD \pm 2 in control corneas (19). A similar study used Orbscan for pachymetry and curvature analysis. In this study, the difference between the central and peripheral corneal thicknesses was measured and in LASIK eyes, and the differences were highly found outside $S D \pm 2$ (20). Using Orbscan method for measuring the curvature difference between 3 and $7 \mathrm{~mm}$ zone, Hick et al. (2I) found $90 \%$ sensitivity and $92.5 \%$ specificity to diagnose LASIK corneas.

In a case-control study, Mootha et al. (9) compared the donor corneas undergone LASIK with normal control group using biomicroscopy, specular microscopy, and light microscopy. In corneas undergone LASIK, they found abnormalities at various degrees at the edge of the flap. Another finding specific to corneas with LASIK was stromal particles with high reflectivity. The authors suggested that the presence of these findings could be used to distinguish corneas undergone LASIK as a screening method.

Another method of distinguishing corneas with LASIK is the use of OCT. The presence of the flap-stroma interface of patients with LASIK can be demonstrated by this technology (22). Wolf et al. in a case study reported OCT scan of a person's cornea who had undergone LASIK 9 months before its death. On the $21^{\text {st }}$ day of organ culture, flap interface differences became clearer and were most prominent on days I-3 and 9-12 (23). Lin et al. (24) compared 24 control donor corneas with 5 LASIK corneas. A decrease in anterior surface curvature was observed in the corneas with LASIK. Furthermore, the LASIK flap was not obvious in the OCT scan. In the in vitro model developed by Prilinger et al., (25) LASIK flaps are formed in organ culture and compared with tissue samples with LASIK history. In both groups, the interface findings were similar in OCT scans.

The eye bank medical standards and practices vary from country to country and sometimes differences may be seen in the same country.

In Turkey, eye banks and corneal transplantation guideline were accepted in 200I and 20 I0; it was decided that medical standards should be determined by the scientific advisory board. However, there are differences in the applications of cornea banks and it is obvious that minimum medical standards for banks are required $(26,27)$.

\section{Conclusion}

The popularity of refractive surgery has increased the chance of encountering donor corneas that have undergone this surgery. Only with a slit-lamp microscope, it is not simple to distinguish postmortem corneas that have undergone refractive surgical operations, mainly PRK and LASIK. Since these tissues may cause intraoperative complications and decrease postoperative refractive outcomes, many different methods 
have been applied to distinguish the tissues in recent years. In endothelial transplantation, donor tissues containing anterior stromal disorder are well tolerated, and endothelial transplantation rate is increasing day by day. Since there is less stromal tissue in the graft used in DMEK, donors with refractive surgery can be used in these operations without additional complications. More frequent use of endothelial keratoplasty will allow corneas that have undergone refractive surgery to be used more as donors.

\section{Disclosures}

Peer-review: Externally peer-reviewed.

Conflict of Interest: None declared.

Authorship Contributions: Involved in design and conduct of the study (EA, BA); preparation and review of the study (EA, BA); data collection (EA, MG, YK); and statistical analysis (BA, MG, YK).

\section{References}

I. Resnikoff S, Pascolini D, Etya'ale D, Kocur I, Pararajasegaram R, Pokharel GP, et al. Global data on visual impairment in the year 2002. Bull World Health Organ 2004;82:844-5I.

2. Pascolini D, Mariotti SP. Global estimates of visual impairment: 2010. Br J Ophthalmol 2012;96:6|4-8.

3. Farge EJ. Eye banking: 1944 to the present. SurvOphthalmol 1989;33:260-3.

4. Demirsoy N, Yilmaz A. Significance of Corneal Transplantation Interms of Medicine, Ethics and Law: Review [Article in Turkish]. TürkiyeKlinikleri J Ophthalmol 2012;21: I7I-83.

5. Saeed AM, Abdrabbo MA. LASIK as an alternative line to treat noncompliant esotropic children. ClinOphthalmol 201 I;5:I795-80I.

6. Fargione RA, Channa P. Cornea donors who have had prior refractive surgery: data from the Eye Bank Association of America. CurrOpinOphthalmol 2016;27:323-6.

7. Patel SV, Erie JC, McLaren JW, Bourne WM. Confocal microscopy changes in epithelial and stromal thickness up to 7 years after LASIK and photorefractive keratectomy for myopia. J Refract Surg 2007;23:385-92.

8. Pisella PJ, Auzerie O, Bokobza Y, Debbasch C, Baudouin C. Evaluation of corneal stromal changes in vivo after laser in situ keratomileusis with confocal microscopy. Ophthalmology 200I; 108: 1744-50.

9. Mootha VV, Dawson D, Kumar A, Gleiser J, Qualls C, Albert DM. Slitlamp, specular, and light microscopic findings of human donor corneas after laser-assisted in situ keratomileusis. Arch Ophthalmol 2004; I22:686-92.

10. Michaeli-Cohen A, Lambert AC, Coloma F, Rootman DS. Two cases of a penetrating keratoplasty with tissue from a donor who had undergone LASIK surgery. Cornea 2002;2I: I I I-3.

II. Farias RJ, Parolim A, Sousa LB. Corneal transplant utilizing a corneal graft that had undergone laser surgery-case report. Arq Bras Oftalmol 2005;68:266-9.

12. Ple-Plakon PA, Shtein RM. Trends in corneal transplantation: in- dications and techniques. CurrOpinOphthalmol 2014;25:300-5.

13. Armour RL, Ousley PJ, Wall J, Hoar K, Stoeger C, Terry MA. Endothelial keratoplasty using donor tissue not suitable for fullthickness penetrating keratoplasty. Cornea 2007;26:5 I5-9.

14. Phillips PM, Terry MA, Shamie N, Chen ES, Hoar KL, Stoeger $C$, et al. Descemet's stripping automated endothelial keratoplasty (DSAEK) using corneal donor tissue not acceptable for use in penetrating keratoplasty as a result of anterior stromal scars, pterygia, and previous corneal refractive surgical procedures. Cornea 2009;28:87I-6.

15. Khalifa YM, Davis D, Mamalis N, Moshirfar M. Donor cornea tissue with prior radial keratotomy: is it suitable for Descemet stripping automated endothelial keratoplasty? Cornea 201 I;30:106I-2.

16. Moshirfar M, Khalifa YM, Davis D, Fenzi CR, Espandar L, Chang $\mathrm{JC}$ et al. Descemet stripping automated endothelial keratoplasty using donor corneas with previous laser in situ keratomileusis or photorefractive keratectomy: a case series and donor cap histopathology. Cornea 2012;31:533-7.

17. Kang SJ, Schmack I, Edelhauser HF, Grossniklaus HE. Donor corneas misidentified with prior laser in situ keratomileusis. Cornea 2010;29:670-3.

18. Terry MA, Ousley PJ. New screening methods for donor eyebank eyes. Cornea 1999; 18:430-6.

19. Ousley PJ, Terry MA. Use of a portable topography machine for screening donor tissue for prior refractive surgery. Cornea 2002;2I:745-50.

20. Ousley PJ, Terry MA. Objective screening methods for prior refractive surgery in donor tissue. Cornea 2002;2I:I8I-8.

2I. Hick S, Laliberté JF, Meunier J, Ousley PJ, Terry MA, Brunette I. Topographic screening of donor eyes for previous refractive surgery. J Cataract Refract Surg 2006; 32:309-17.

22. Ustundag C, Bahcecioglu H, Ozdamar A, Aras C, Yıldırım R, Özkan S. Optical coherence tomography for evaluation of anatomical changes in the cornea after laser in situ keratomileusis. J Cataract Refract Surg 2000;26: |458-62.

23. Wolf AH, Neubauer AS, Priglinger SG, Kampik A, WelgeLuessen UC. Detection of laser in situ keratomileusis in a postmortem eye using optical coherence tomography. J Cataract Refract Surg 2004;30:49I-5.

24. Lin RC, Li Y, Tang M, McLain M, Rollins AM, lzatt JA, Huang D. Screening for previous refractive surgery in eye bank corneas by using optical coherence tomography. Cornea 2007;26:594-9.

25. Priglinger SG, Neubauer AS, May CA, Alge CS, Wolf $A H$, Mueller A, et al. Optical coherence tomography for the detection of laser in situ keratomileusis in donor corneas. Cornea 2003;22:46-50.

26. Şencan S. History and Development of Eye Banking in Turkey [Article in Turkish]. TürkiyeKlinikleri J Ophthalmol Special Topics 2016;9:4-22.

27. Gain P, Jullienne R, He Z, Aldossary M, Acquart S, Cognasse F, et al. Global Survey of Corneal Transplantation and Eye Banking. JAMA Ophthalmol 2016;134:167-73. 